Urologe 2013 $52: 7-8$

DOI 10.1007/s00120-012-3073-5

Online publiziert: 6 . Januar 2013

(c) Springer-Verlag Berlin Heidelberg 2013

R. Stein ${ }^{1} \cdot$ J. Steffens ${ }^{2}$

${ }^{1}$ Abteilung Kinderurologie, Urologische Klinik und Poliklinik, Universitätsmedizin, Johannes Gutenberg-Universität Mainz, Mainz

${ }^{2}$ Klinik für Urologie und Kinderurologie, St. Antonius-Hospital Eschweiler, Eschweiler

\title{
Kinderurologie im Wandel der Zeit
}

ren Betreuung der Kinder. Ebenso wichtig ist die Zusammenarbeit der einzelnen Kliniken, insbesondere, wenn es um die Weiterbildung geht. Diese kann häufig nicht nur an einer Klinik erfolgen und ein Wechsel der Ausbildungsstätten bringt hier eine deutliche Erweiterung des Erfahrungshorizonts. Die gemeinsamen Artikel verschiedener Kliniken tragen diesem Anspruch hier Rechnung.

Die Harnwegsinfektionen (HWI) gehören zu den häufigsten bakteriellen Infektionen im Kindesalter und erfordern eine exakte mikrobiologische Diagnostik. Beetz u. Wagenlehner nehmen kritisch zu den neusten Studien und Leitlinien Stellung. Die Autoren geben nach Auswertung teils überraschender neuer Studienergebnisse und Darstellung bewährter und neu verfügbarer Medikamente eine aktuelle Therapieempfehlung. Ein wesentliches Ziel der weiterführenden Diagnostik ist die Vermeidung irreversibler Nierenparenchymschäden.

Seibold und Mitarbeiter präsentieren unter dem Symptom Bettnässen das häufige Krankheitsbild der monosymptomatischen Enuresis. Sie weisen auf die Notwendigkeit einer einheitlichen Terminologie zur exakten Klassifikation hin. Streng abzugrenzen ist monosymptomatische Enuresis von der kindlichen Harninkontinenz. Eine nicht-invasive Diagnostik ist ausreichend. Die Behandlungssäulen sind die Urotherapie, die Desmopressinsowie die Alarmtherapie und deren Kombination.

Geyer und Mitarbeiter stellen das heterogene Krankheitsbild der Harnblasen- funktionsstörungen dar. Harnspeicherund Harnblasenentleerungsphase können betroffen sein. Nicht-neurogene Störungen lassen sich durch nicht-invasive Diagnostik erkennen und konservativ behandeln. Bei neurogenen Erkrankungen ist eine frühzeitige, invasive Diagnostik notwendig, um eine multimodale konservative und evtl. operative Therapie rechtzeitig zur Verbesserung der Lebensqualität und Erhalt der Nierenfunktion einleiten zu können.

Anheuser und Mitarbeiter berichten über den primären Megaureter, der in 80\% der Fälle spontan mit dem Längenwachstum maturiert. Eine antibiotische Reinfektprophylaxe ist nur im ersten Lebensjahr bei erhöhtem Pyelonephritisrisiko notwendig. Eine Operationsindikation besteht bei symptomatischen und obstruktiv-refluxiven Formen mit szintigraphisch nachweisbarer Obstruktion.

Die kontroversen Diskussionen zwischen diagnostischem Nihilismus und invasiver Therapie beim Reflux werden von Stein und Mitarbeitern dargestellt. Gemeinsames Ziel aller derzeit konkurrierenden diagnostischen Strategien und Therapieansätzen ist es, pyelonephritische Nierenschädigungen mit dem wirksamsten und gleichzeitig am wenigsten belastenden Konzept zu verhindern. Die dargestellten Behandlungsstrategien berücksichtigen neben Alter und Geschlecht, das Vorhandensein dysplastischer oder pyelonephritischer Nierenparenchymdefekte, Refluxgrad, die klinische Symptomatik, Blasenfunktionsstörungen und die Häufigkeit bzw. schwere rezidivieren- stellung der einzelnen Artikel bezeugt die Notwendigkeit einer interdisziplinä- 
de HWI als Kriterien für die Therapieentscheidung.

Die Therapie der jugendlichen und adulten Varikozele wird teilweise sehr kontrovers diskutiert. Zur Behandlung der jugendlichen Varikozele liegen deutlich weniger Daten vor. Nach einer kritischen Bewertung der vorhandenen Studienlage kommen Kliesch, Czeloth u. Kälble zu dem Schluss, dass die Indikation zur Therapie einer Varikozele beim Jugendlichen derzeit nur individuell und sehr zurückhalten gestellt werden sollte. Trotz des relativ häufigen Befunds einer Varikozele im Jugendalter, gibt es nur sehr wenige Studien, die endokrine Marker (z. B. FSH/Inhibin B), Ejakulatparameter oder induzierte Schwangerschaften untersuchten. In dem Beitrag wird dargestellt, ob die durch eine Operation bewirkte Verbesserung des Hodenvolumens und eine Abnahme der Hodenvolumendifferenz mehr als ein kosmetischer Faktor ist. Zumindest werden die mit einer Varikozele möglicherweise einhergehenden Schmerzen durch eine operative oder radiologische Intervention günstig beeinflusst.

Die Nephrolithiasis im Kindesalter ist in den deutschsprachigen Regionen selten. Knoll u. Humke geben eine aktuelle Übersicht zu diesem Krankheitsbild. Kindliche Harnsteine sind meist Folge metabolischer Störungen und gelegentlich anatomischer Harntransportsdefekte. Entscheidend ist eine Steinanalyse und erweiterte Stoffwechseldiagnostik. Behandlungsziele sind vollständige Steinfreiheit, Prävention von Steinrezidiven und Korrektur anatomischer und metabolischer Ursachen. Die extrakorporale Stoßwellenlithotripsie (ESWL) erzielt im Kindesalter bessere Ergebnisse als im Erwachsenenalter. Miniaturisierte Endoskope ermöglichen heutzutage eine erfolgreiche transureterale oder perkutane Steinsanierung.

Wir erhoffen uns mit der Darstellung dieser teils sehr kontroversen, jedoch im täglichen Umgang mit kinderurologischen Patienten, häufigen Problemen einen kleinen Einblick in die Kinderurologie gegeben und so das Interesse geweckt zu haben. Die teils sehr ausführlichen Literaturverzeichnisse erlauben dem Interessierten eine umfassende Aktualisierung vorhandenen Wissens.

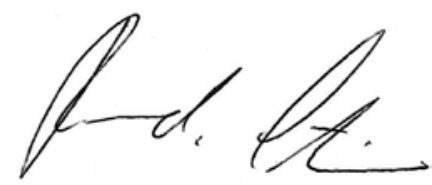

R. Stein

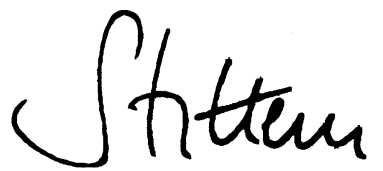

J. Steffens

\section{Korrespondenzadressen}

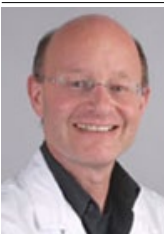

Prof. Dr. R. Stein

Abteilung Kinderurologie, Urologische Klinik und Poliklinik, Universitätsmedizin, Johannes GutenbergUniversität Mainz, Langenbeckstraße 1, 55131 Mainz Raimund.Stein@ unimedizin-mainz.de

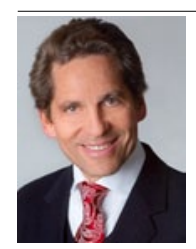

Prof. Dr. J. Steffens

Klinik für Urologie und Kinderurologie, St. Antonius-Hospital Eschweiler,

Dechant-Deckers-Straße 8, 52249 Eschweiler joachim.steffens@ sah-eschweilder.de

\section{Deutsche Gesellschaft für Chirurgie positioniert sich zu Transplantationschirurgie}

Um die Situation der Organspende in Deutschland im Sinne der Patienten zu stärken, hat die Deutsche Gesellschaft für Chirurgie (DGCH) Empfehlungen zur Transplantationschirurgie erarbeitet. Unter www.dgch.de kann die vollständige Stellungnahme öffentlich eingesehen werden. In dem 10 Punkte umfassenden Papier thematisiert die DGCH unter anderem Indikation und Dokumentation von Transplantationen und die Rolle von Transplantationsregistern, -konferenzen und -zentren. Die Fachgesellschaft verurteilt jeglichen Verstoß gegen die medizinischethischen Grundsätze und Regeln des Transplantationsgesetzes und der Richtlinien der Bundesärztekammer. So darf die Entscheidung für eine Transplantation und die Aufnahme in eine Warteliste ausschließlich nach medizinischen Kriterien gefällt werden. In diesem Zusammenhang lehnt die DGCH auch Bonuszahlungen für gesteigerte Transplantationszahlen entschieden als ethisch nicht vertretbar ab. Außerdem plädiert die Fachgesellschaft für eine Konzentration der Transplantationsmedizin auf wenige Zentren. Die Ergebnisse und Qualitätskennzahlen dieser Zentren sollten zudem öffentlich zugänglich gemacht werden. Ein Schritt zu mehr Transparenz sei auch ein zentrales Transplantationsregister. Hierfür gelte es, zügig Finanzierung und Datenschutz zu regeln, erklärt die DGCH. Des Weiteren unterstützt die DGCH in ihrer Stellungnahme ausdrücklich die Aufarbeitung und Untersuchung aller Verdachtsfälle. Die aktuelle Debatte dürfe nicht dazu führen, dass ein für viele Patienten lebensrettendes Verfahren Schaden nehme.

Quelle: Deutsche Gesellschaft für Chirurgie (DGCH), Stuttgart www.dgch.de 